\title{
EFFECTS OF [10]-GINGEROL IN COMBINATION WITH DOXORUBICIN ON TRIPLE NEGATIVE BREAST CANCER IN VITRO AND IN VIVO
}

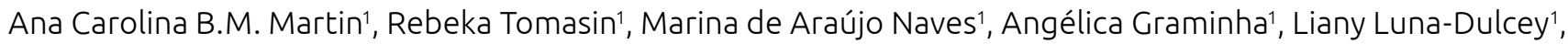
Ramon H.G. Teles ${ }^{1}$, Márcia Regina Cominetti ${ }^{1}$

${ }^{1}$ Laboratory of Biology of Aging, Department of Gerontology, Universidade Federal de São Carlos - São Carlos (SP), Brazil.

Objective: Triple negative breast cancer (TNBC) often metastasizes to bones, lungs and brain. It does not respond to current target therapies, and thus, limited treatments are available, especially for late stage disease. We have previously shown that [10]- gingerol has cytotoxic and anti-metastatic properties against TNBC in vivo and in vitro. To further mimic a clinically relevant scenario, which often involves the use of multiple drugs, we evaluated the effects of [10]-gingerol in combination with doxorubicin for TNBC treatment. Methods: TNBC cells (MDA-MB-231 and 4T1Br4) were treated with [10]-gingerol (10G), doxorubicin (DOX) or both (10G+DOX) and assayed for viability, colony formation and apoptosis. Next, TNBC cells were inoculated orthotopically into the mammary fat pad of female mice. When tumours became palpable, mice were treated with saline solution (Control), 10G (5-10 mg $/ \mathrm{kg}$ daily), DOX (3mg/ $/ \mathrm{kg}$ twice a week) or a combination of both (10G xmg+DOX xmg). Body weight and primary tumour growth were monitored throughout the entire experiment (30 days). Upon euthanasia, blood was collected to analyse the presence of circulating tumour cells (CTCs), as well as toxicity markers. Lungs, bones and brain were harvested for metastatic burden assessment. Results: The association of $10 \mathrm{G}$ and DOX had additive inhibitory effects on viability and colony formation and increased apoptosis in TNBC cells. Regarding to the in vivo experiments, $10 \mathrm{G}$, especially when combined with DOX, dramatically reduced primary tumour growth, as well as the incidence and/or the number of viable circulating tumour cells. As a result, metastasis was also impaired. Moreover, the combination (10G+DOX) not only had the aforementioned additive antitumour and antimetastatic effects, but also attenuated doxorubicin-induced toxicity. Remarkably, only 7/15 mice treated with DOX remained alive at endpoint, and this number increased to $11-12 / 15$ when mice received $10 \mathrm{G}$ alongside ( 5 or $10 \mathrm{mg} / \mathrm{kg}$, ip, daily, respectively). We also noticed attenuation in weight loss and hepatotoxicity (AST and ALT levels in serum) when the combination regimen was used. Additional analysis to elucidate the molecular mechanisms involved in these effects are ongoing. Conclusion: Taken together, our data indicate that [10]-gingerol could be used as adjuvant/complementary therapy for TNBC, enhancing the anticancer activity and attenuating undesired side effects of conventional chemotherapeutic agents. 